\title{
Challenges and countermeasures of thoracic oncology in the epidemic of COVID-19
}

\author{
Haoyue Guo ${ }^{1,2}$, Xiaoxia Chen ${ }^{1}$, Chunxia $\mathrm{Su}^{1}$, Yu Liu ${ }^{1,2}$, Hao Wang ${ }^{1,2}$, Chenglong Sun ${ }^{1,2}$, Peixin Chen ${ }^{1,2}$, \\ Minlin Jiang ${ }^{1,2}$, Yi Xu ${ }^{1,2}$, Shengyu Wu ${ }^{1,2}$, Keyi Jia ${ }^{1,2}$, Sha Zhao ${ }^{1}$, Wei Li ${ }^{1}$, Bin Chen ${ }^{1}$, Lei Wang ${ }^{1}, J_{i a} \mathbf{Y u}^{1}$, \\ Anwen Xiong ${ }^{1}$, Guanghui Gao ${ }^{1}$, Fengying $\mathrm{Wu}^{1}$, Jiayu $\mathrm{Li}^{1}$, Lingyun $\mathrm{Ye}^{1}$, Bing Bo ${ }^{1}$, Shen $\mathrm{Chen}^{1}$, Shengxiang \\ Ren $^{1}$, Yayi He ${ }^{1}$, Caicun Zhou ${ }^{1}$
}

${ }^{1}$ Department of Medical Oncology, Shanghai Pulmonary Hospital, Tongji University Medical School Cancer Institute, Tongji University School of Medicine, Shanghai 200433, China; ${ }^{2}$ Tongji University, Shanghai 200433, China

Contributions: (I) Conception and design: H Guo, Y He; (II) Administrative support: C Su, S Ren, C Zhou; (III) Provision of study materials or patients: S Wu, K Jia, C Sun, L Ye, B Bo; (IV) Collection and assembly of data: H Guo, Y Liu, H Wang, P Chen, M Jiang, Y Xu, A Xiong, G Gao, F Wu, J Li; (V) Data analysis and interpretation: H Guo, S Zhao, W Li, B Chen, L Wang; (VI) Manuscript writing: All authors; (VII) Final approval of manuscript: All authors.

Correspondence to: Yayi He; Shengxiang Ren; Caicun Zhou. Department of Medical Oncology, Shanghai Pulmonary Hospital, Tongji University Medical School Cancer Institute, Tongji University School of Medicine, No. 507 Zhengmin Road, Shanghai 200433, China.

Email: 2250601@qq.com; harry_ren@126.com; caicunzhoudr@163.com.

\begin{abstract}
Since December, 2019, a 2019 novel coronavirus disease (COVID-19) infected by the severe acute respiratory syndrome corona virus 2 (SARS-CoV-2) emerged in Wuhan, Hubei province, and the epidemic situation has continued to spread globally. The epidemic spread of COVID-19 has brought great challenges to the clinical practice of thoracic oncology. Outpatient clinics need to strengthen the differential diagnosis of initial symptoms, pulmonary ground-glass opacity (GGO), consolidation, interstitial and/or interlobular septal thickening, and crazy paving appearance. In the routine of oncology, the differential diagnosis of adverse events from COVID-19 is also significant, including radiation pneumonitis, checkpoint inhibitor pneumonitis (CIP), neutropenic fever, and so on. During the epidemic, indications of transbronchial biopsy (TBB) and CT-guided percutaneous thoracic biopsy are strictly controlled. For patients who are planning to undergo biopsy operation, screening to exclude the possibility of COVID-19 should be carried out. For confirmed or suspected patients, three-level protection should be performed during the operation. Disinfection and isolation measures should be strictly carried out during the operation. At last, more attention to the protection of cancer patients and give priority to the treatment of infected cancer patients.
\end{abstract}

Keywords: 2019 novel coronavirus disease (COVID-19); severe acute respiratory syndrome corona virus 2 (SARS-CoV-2); thoracic oncology

Submitted Feb 17, 2020. Accepted for publication Mar 18, 2020.

doi: $10.21037 /$ tlcr.2020.02.10

View this article at: http://dx.doi.org/10.21037/tlcr.2020.02.10

\section{Introduction}

In December, 2019, a pneumonia named as 2019 novel coronavirus disease (COVID-19) caused by the severe acute respiratory syndrome corona virus 2 (SARS-CoV-2) outbroke in Wuhan, Hubei province, China and then spread to multiple countries around the world (1). Coronaviruses can attack multiple systems in a variety of animals and primarily the respiratory tract in humans (2).

After up to 3-to-14-day incubation period, most patients with COVID-19 initially had fever, cough and shortness of breath. Additional systemic symptoms were muscle pain, 
headache, confusion, chest pain, and diarrhea (3). As the progression of the disease and the conduction of related medical operations, some patients started to face lifethreatening multiple organ dysfunction, including acute respiratory injury, acute respiratory distress syndrome (ARDS), acute myocarditis, severe hepatitis, acute renal injury, septic shock, and so on (3).

According to the latest treatment plan proposed by National Health Commission of the People's Republic of China, the epidemiological characteristics of COVID-19 include following points: (I) the source of infection is the clinical and asymptomatic patients of COVID-19; (II) human-to-human transmission is confirmed via respiratory droplets and direct or indirect contact with infected secretions. Other transmission routes such as aerosol and digestive tract have not yet been defined; (III) the general population is susceptible. The clinically diagnosed cases of COVID-19 in Hubei province can be confirmed by two of the clinical manifestations with imaging features of pneumonia, where the clinical manifestations include fever and(or) respiratory symptoms, and decreased or normal WBC count, or decreased lymphocyte count. Furthermore, the diagnosis of confirmed cases outside Hubei province requires the following etiological evidence: the nucleic acids of SARS-CoV-2 can be detected in respiratory specimens or blood samples via real-time polymerase chain reaction (RT-PCR) or the virus detected in respiratory specimens or blood samples is exceedingly homologous to known SARS-CoV-2 by viral gene sequencing. Therefore, the epidemic spread of COVID-19 has posed great challenges to the clinical practice of thoracic oncologists.

\section{Differential diagnosis with other lung diseases}

All outpatients should be routinely inquired about epidemiological history including whether traveled to Wuhan and surrounding areas or other communities with medical records, whether contacted the person who had traveled to Wuhan and surrounding areas or other communities with medical records, whether contacted the person who had respiratory symptoms, whether had fever, cough or shortness of breath in the past 2 weeks, whether there was an agglomerative disease and whether have the history of wildlife exposure. In addition to the epidemiological history, the differential diagnosis of COVID-19 with other lung diseases can be considered based on following clinical symptoms and signs.

\section{Fever, respiratory symptoms and decreased white blood cells (WBC) or lymphocytes}

Fever, respiratory symptoms such as nonproductive cough and shortness of breath, and decreased WBC are the most common first symptoms and signs of COVID-19 (3), which are also the most vital clinical evidence except radiological images. These signs and symptoms are also found in various kinds of pneumonia, while lung cancers patients face a higher incidence of pneumonia at $50-70 \%$ (4), due to the weak immune system and the damage of lung architecture. Importantly, the WBC count or lymphocyte count in COVID-19 can reveal some excluded clues that, in most cases of bacterial pneumonia, the WBC count rises, though it may decrease in severe cases $(5,6)$. Meanwhile, if the pneumonia patient shows a decreased WBC count with lower lymphocyte count, Mycoplasma pneumoniae pneumonia (MP) or viral pneumonia is supposed to be considered (6,7). MP can be accurately diagnosed via the etiologic results based on throat swab culture or detection of IgM or IgA antibodies in peripheral blood (8). As for the differentiation of COVID-19 from other common viral pneumonia, we can consider based on epidemiological characteristics and different clinical symptoms (Table 1). At last, the microbiological diagnosis based on the detection of viral antigens in respiratory tract specimens, via culture and immunofluorescence microscopy, is the diagnostic gold standard (6). Currently, the introduction of RT-PCR has enhanced the detective ability of respiratory viruses, which has been one of the most important tools to help us determine whether viral pneumonia is caused by SARS$\mathrm{CoV}-2$ (9). Recently, an IgM antibody against SARS-CoV$2 \mathrm{~g}$ is also about to be put into clinical practice, which is promising to become a rapid diagnostic method for COVID-19.

\section{Pulmonary ground-glass opacity (GGO) or ground-glass nodule (GGN)}

GGO is defined as a field of faint opacity with the capability of showing pulmonary vessels or bronchial structures, indicating multiple diseases, including inflammation, pulmonary fibrosis, alveolar hemorrhage or neoplasm $(10,11)$. The CT imaging of COVID-19 at early stages is usually presented as single or multiple GGO nodules (12), patchy shadows, or flaky shadows, distributed in the $1 / 3$ of outer lung field and subpleural space. As a result, the CT image of early COVID-19 has some similarities 


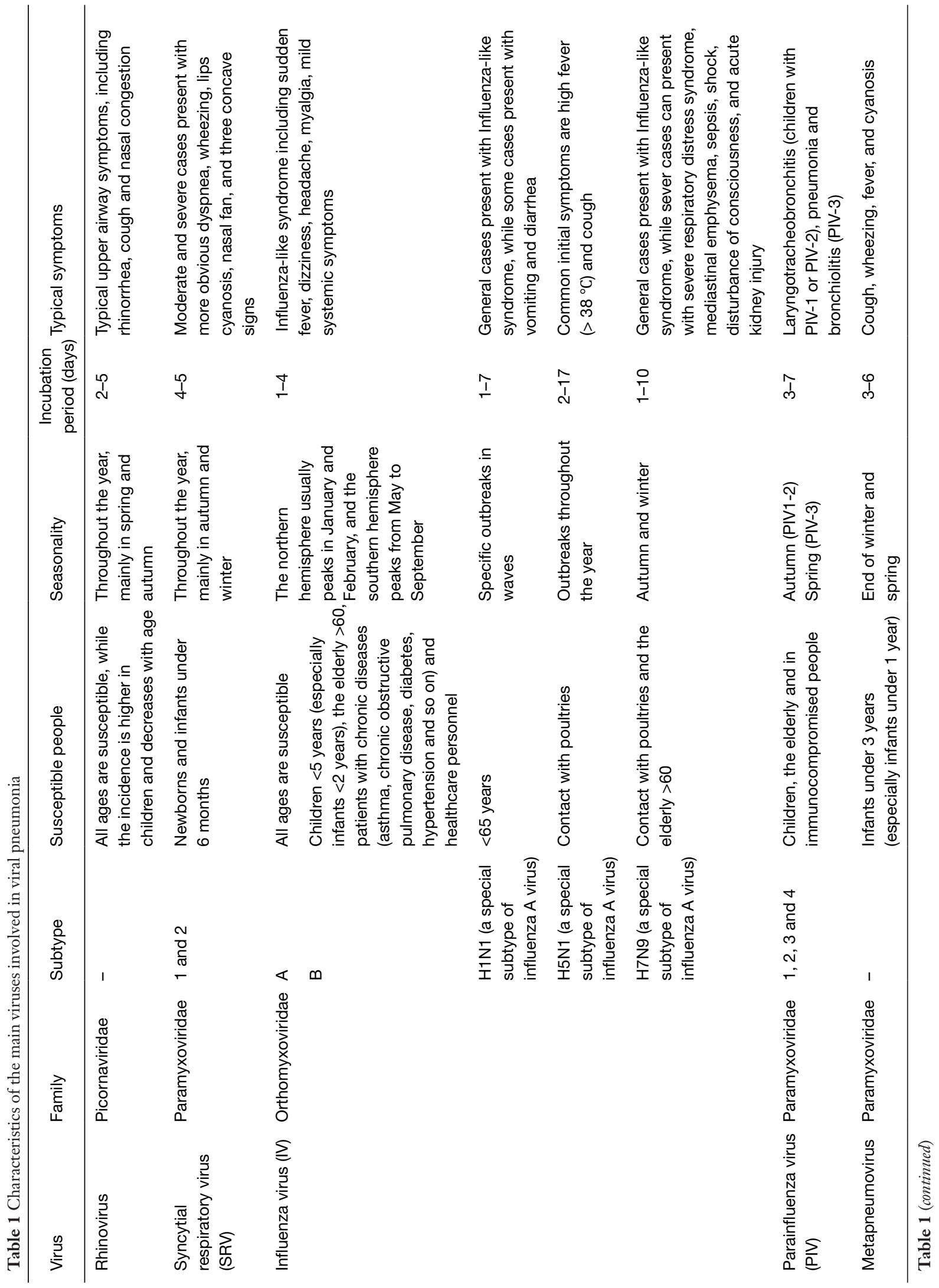




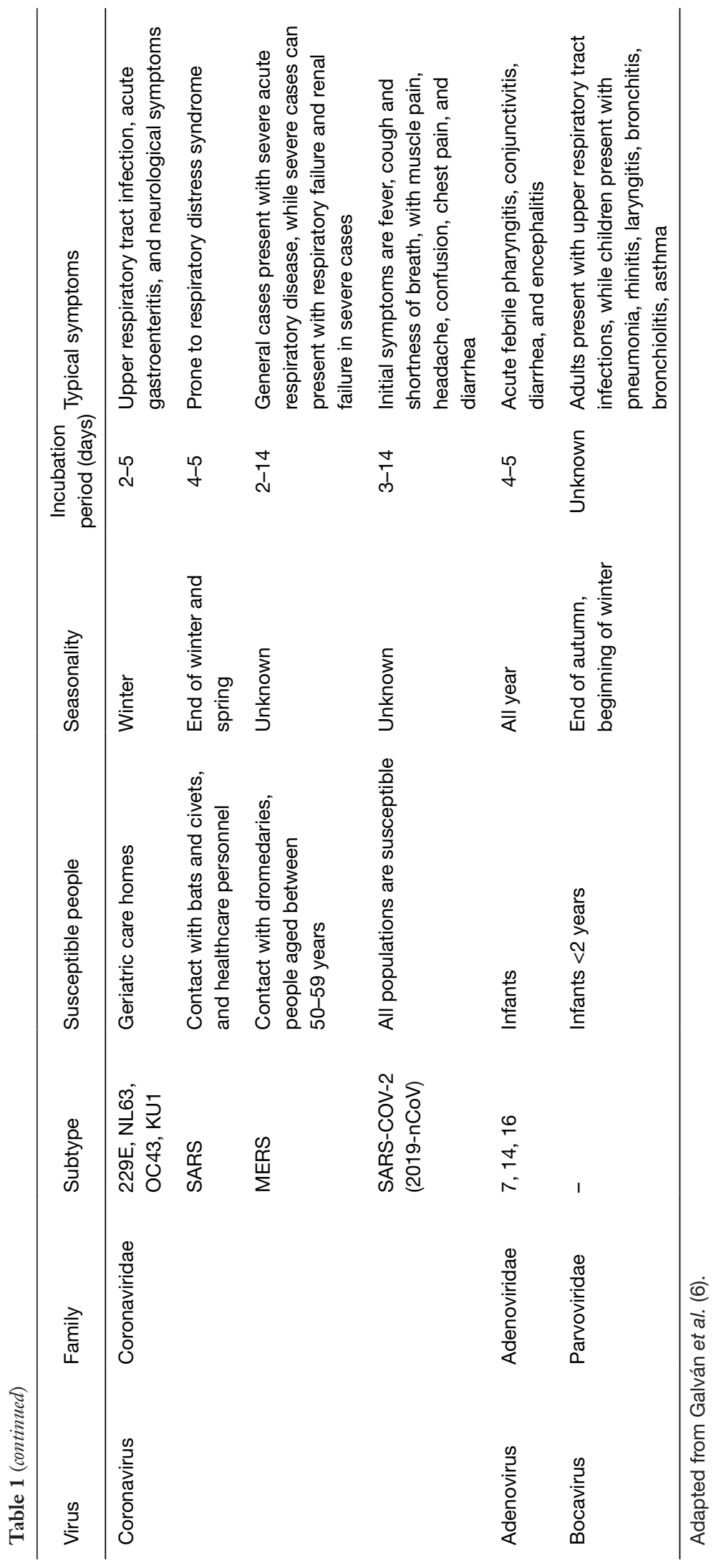


with that of GGN in lung cancer. Although GGO is one of the most important radiologic evidence for early lung cancers, compared with COVID-19, the lesions of GGN have more uniform density and clearer boundaries, without distribution characteristics, and may be stable after 2- to 3-month follow-up (11). Considering these distinctions, follow-up observations is indispensable for the patients with fist-occurring GGN to avoid misdiagnosing COVID-19 as early-stage lung cancer.

\section{Pulmonary consolidation}

As the disease progresses, pulmonary consolidation occurs in most of the COVID-19 patients (13). A pulmonary consolidation refers as a region of normally compressible lung tissue with reduced alveolar air content and denser lung texture, which is a nonspecific sign but most often in bacterial or organizing pneumonia. However, it is well recognized that lung cancer can present as parenchymal infiltration mimicking the imaging features of pneumonia especially pulmonary consolidation, also known as pneumonic-type lung cancer (PTLC), which may be misdiagnosed as inflammatory lung diseases, delaying accurate diagnosis (14). Although the imaging of PTLC is highly similar to that of pneumonia, the distribution of lesions in PTLC is more limited. Most of the PTLC is faint and flaky GGO, with nodules or bronchial stiffness in the consolidation area $(15,16)$. When radiology cannot provide indiscriminate evidence, cancer imaging of ${ }^{18} \mathrm{~F}$-fluorodeoxyglucose single-photon-emission computer tomography $\left({ }^{18} \mathrm{~F}-\mathrm{FDG}\right.$-SPECT $)$, transbronchial biopsy (TBB) and percutaneous needle aspiration can improve the accuracy of diagnosis.

\section{Interstitial and/or interlobular septal thickening}

Interstitial and/or interlobular septal thickening is reported as the chest CT feature in 75\% patients of COVID-19 (13), which is also the typical appearance of pulmonary lymphangitic carcinomatosis (PLC). The interlobular septal thickening in PLC is most often nodular and irregular, which is diverse from COVID-19 $(17,18)$. Second, almost all PLC present beaded thickening of bronchovascular bundles and some present with unilateral or bilateral hilar lymphadenopathy, which is also much rare in COVID-19 (19). Considering the difference in tumor history and radiologic features, the discrimination between PLC and COVID-19 can be carried out.

\section{Crazy paving appearance}

According the emerging reports of COVID-19, the chest CT of some patients presented with crazy paving appearance (20), defined as a sign on chest high resolution computed tomography (HRCT), where GGO appears with interstitial and/or interlobular septal thickening (21). However, in COVID-19, crazy paving appearance occurs considerably less frequently than the GGO and interstitial and/or interlobular septal thickening appearing separately. In contrast, it is much more common in other conditions such as acute interstitial pneumonia (AIP), pulmonary alveolar proteinosis (PAP), ARDS and bacterial pneumonia (21), among which AIP is relatively more difficult to be diagnosed. In the early stage of AIP, the radiographic images can demonstrate GGO in the lateral field of lung. When the disease progresses rapidly, the imaging can show the transformation of consolidation and fibrosis in 1 week (22), which is rarely seen especially in the early stages of COVID-19. Moreover, lung biopsy and etiological results can help establish a differential diagnosis, among which the etiological detection is the most reliable method to distinguish AIP from COVID-19 (22). As for suspected patients of PAP, the diagnosis is usually established by bronchoalveolar lavage (BAL) fluid and/ or lung biopsy (gold standard). BAL fluid of most cases of PAP contains the periodic acid Schiff positive material (23), which is absent in cases of COVID-19, making it easy to differentiate between these two diseases.

\section{Differential diagnosis with cancer complications and treatment side effects}

\section{Radiation pneumonitis}

Radiation pneumonitis is a kind of pulmonary inflammation caused by radiation therapy to the chest. The imaging of radiation pneumonitis often located in radiation fields, with fever, shortness of breath and dry cough, which shares similar symptoms and imaging with COVID-19 (24). Except of the radiation history, there are four extra differences between radiation pneumonitis and COVID-19. First, the symptoms of radiation pneumonitis mainly occur within 3 to 12 weeks after radiotherapy (25), which is longer than the incubation period of COVID-19. Second, in addition of respiratory symptoms, crackles, erythema or hyperpigmentation are also found on the skin that has undergone radiotherapy (25). Compared with radiation pneumonitis, skin system symptoms are currently much 
less reported in COVID-19. Third, radiation pneumonitis happens with modest increases in WBC count (25), which is contrary from COVID-19. Forth, the radiographic lesions of radiation pneumonitis are more limited than that of COVID-19, as lesions of radiation pneumonitis locate within the field experienced a radiation (25). In view of these differences and etiological detections, it is not difficult to distinguish between these two diseases.

\section{Checkpoint inbibitor pneumonitis (CIP)}

CIP occurs after the immune checkpoints inhibitors (ICIs) therapy, with non-specific respiratory symptoms and new inflammatory lesions on CT, following the exclusion of other reasons (26). CIP can demonstrate diverse radiographic images, among which the most frequent images are similar to that of cryptogenic organizing pneumonia (COP), with GGO or consolidation distributed in peripheral lung field or along per bronchi, followed by nonspecific interstitial pneumonia (NSIP), with GGO and reticular opacities located in the peripheral and lower lungs (27). As mentioned above, GGO, consolidation and interstitial lesions are common radiographic features in COVID-19, therefore, the following two differences are critical to differentiate CIP from COVID-19. First, the history of ICIs therapy is the foundation of CIP. CIP should be considered when new radiographic lesions appeared after ICIs therapy, with or without symptom (28). At last, the negative etiological results of sputum and serum especially deep sputum specimens obtained during bronchoscopy, can give a boundary between CIP and COVID-19 (28).

\section{Lung toxicity of targeted therapy}

Gefitinib, osimertinib and erlotinib are typical inducements of interstitial pneumonitis among targeted therapy agents $(29,30)$. As mentioned above, interstitial pneumonitis shares similar radiographic lesions with COVID-19. Importantly, pneumonias after the targeted therapy have a critical clinical feature, that most cases degenerate when the therapy is suspended and when corticosteroid treatment is started. Also, pneumonias after the targeted therapy can recurrent once restart the targeted therapy. The relationship between the disease progression and the use of drug is an extremely good indication for targeted-therapy-associatedpneumonitis to be distinguished from COVID-19 (31). In addition, interstitial pneumonia after targeted therapy is often sensitive to hormone therapy, and the efficacy of hormone therapy can also be considered as an important differential diagnostic basis (31).

\section{Postobstructive pneumonia}

Postobstructive pneumonia refers to an inflammatory lesion located in the lung parenchyma following bronchial obstruction (32), which often occurs in primary or metastatic lung cancers (33-35), with an evolution from obstructive atelectasis to postobstructive pneumonia (35). Various bacteria, fungi and viruses can induce postobstructive pneumonia, thus, the typical symptoms of it are close to that of any pneumonia (36), including COVID-19. Therefore, focusing on the radical cause of postobstructive pneumonia-airway obstruction, is the key to distinguishing it from COVID-19. Chest CT can help show the location of obstruction and determine whether the distal airway is smooth $(37,38)$. Furthermore, the fiber bronchoscopy is the most intuitive and critical method for diagnosing airway obstruction (39). In conclude, with the above evidence of airway obstruction, symptoms of pneumonia are no longer a barrier to differentiate postobstructive pneumonia from COVID-19.

\section{Cancer-associate bacterial pneumonia}

Cancer itself with its treatment makes cancer patients more susceptible to bacterial pneumonias, because of weaken immune response to respiratory bacteria. Multiple mechanisms work together to increase the susceptibility of cancer patients to bacterial pneumonia, such as the neutropenia after chemotherapy, lung structural damages caused by tumor cells invasion and undernourishment (40-42). Additionally, frequent medical visits expose cancer patients to a variety of rare and resistant bacteria $(42,43)$. Flexible bronchoscopy with BAL is the preferred diagnostic operation for cancer-associate bacterial pneumonia, via seeking etiological evidence. Moreover, serum procalcitonin (PCT) has been confirmed as a diagnostic marker of bacterial infection in solid tumors patients (43). As reported, increased PCT (>1.52 dng/dL) even demonstrated a better sensitivity and specificity for a Gram-negative infection than a Gram-positive infection $(43,44)$. In short, combining the etiologic test of BAL and the value of serum PCT provides a simple and reliable identification method between cancer-associated bacterial pneumonias and COVID-19. 


\section{Fever in cancer patients}

\section{Neutropenic fever induced by chemotherapy}

Chemotherapy often leads to a reduced WBC count, called leukopenia (45). Neutropenia is defined as an absolute neutrophil count $(\mathrm{ANC})<2.0 \times 10^{9} / \mathrm{L}$, while agranulemia refers as ANC $<0.5 \times 10^{9} / \mathrm{L}$ (46). Neutropenic fever(febrile neutropenia) is a single oral temperature over $38.3^{\circ} \mathrm{C}\left(101{ }^{\circ} \mathrm{F}\right)$ or a temperature over $38.0^{\circ} \mathrm{C}\left(100.4^{\circ} \mathrm{F}\right)$ lasting more than 1 hour of neutropenia patients (46), with an incidence of $14 \%$ after chemotherapy (47), which can indicate infection. The main symptoms of neutropenic fever, especially accompanied by cough or other respiratory symptoms are close to those of COVID-19. However, COVID-19 leads to a decreased lymphocyte count instead of neutrophil count, which is a significant difference from neutropenic fever.

\section{Drug fever after chemotherapy}

The definition of drug fever is a fever happens following the application of certain drug and disappears after discontinuing the drug, without other obvious reasons (48). About $3-6 \%$ of adverse drug reactions only present with fever $(48,49)$. Therefore, although in most cases, fever after chemotherapy is considered as an infection with neutropenia, drug fever should be viewed as an important cause (50). Interestingly, a study concluded that the febrile episodes appear on the third or fourth day after chemotherapy are typical adverse drug reactions of chemotherapy, rather than infection (50). Overall, the pathogenic examinations, the relationship between the drug administration with fever, and the time point of fever can help us accurately determine whether the fever after chemotherapy is a drug fever or a secondary infection including COIV-19.

\section{Neoplastic fever}

Neoplastic fever is known as fever caused by cancer itself, which is considered as the most common reason of fever of unknown origin (FUO) in cancer patients (51). Malignant tumors can cause hyperthermia through different mechanisms, including secreting cytokines such as interleukin-2 (IL-2), tumor necrosis factor- $\alpha(\mathrm{TNF}-\alpha)$ and interleukin-6 (IL-6) (52), while tumor necrosis and dead tissue can also release TNF- $\alpha$ and other pyrogens (53). Up to now, there is no research giving a reliable distinction in clinical symptoms between neoplastic fever and infectious fever. However, nonsteroidal anti-inflammatory drugs have a good control of neoplastic fever, which is a good clue to neoplastic fever (54).
Complicated with opportunistic pulmonary infections As mentioned above, cancer patients in an immunosuppressed state are more likely to develop infectious diseases including opportunistic infections like invasive pulmonary aspergillosis (IPA) and pneumocystis pneumoniae (PCP), because of the tumor itself or the tumor treatment. In previous paragraphs, we have illustrated the identification of common lung infections with COVID-19, including viral pneumonia and bacterial pneumonia. So, we also offer a differential diagnosis of opportunistic infections in cancer patients with COVID-19.

About $1-8 \%$ of patients with solid tumors suffer from IPA $(55,56)$. The typical imaging feature of IPA is a focal infiltrate of nodules (57), which is dramatically distinctive from that of COVID-19. Moreover, microbiologic or histopathologic confirmation in biopsy specimens offers a clear definition (57).

The clinical symptoms of PCP in cancer patients present with fever, nonproductive cough, tachypnea, and hypoxemia, in a very short time, which is highly similar to that of COVID-19. The images on chest X-ray typically demonstrate diffuse interstitial lesions or lobar infiltrates (57). When radiology shows diffuse interstitial lesions, it's hard to differentiate PCP from COVID-19 based on symptoms and imaging. In this case, the detection of cysts or trophozoites in respiratory specimens, by sputum induction or BAL can facilitate the discrimination (58).

\section{Screening before TBB and CT-guided percutaneous thoracic biopsy}

TBB and CT-guided percutaneous thoracic biopsy are most common and important methods for diagnosing cancers. During the epidemic period of COVID-19, patients who meet indications without contraindications, require TBB or CT-guided percutaneous thoracic biopsy should undergo preliminary screening. Preliminary screening of COVID-19 includes body temperature, related epidemiological history, pulmonary CT, blood routine, C-reactive protein, PCT, and the detection of respiratory pathogens in respiratory specimens or blood samples including influenza virus, chlamydia pneumoniae, mycoplasma pneumoniae, respiratory syncytial virus, adenovirus, and coxsackie virus. When pulmonary CT presents multiple patchy shadows and interstitial lesions, nucleic acid test of SARS-CoV-2 is supposed to be performed to confirm the diagnosis. In view of the high probability of false negative in single test even if the first test is negative, medical staff should also be cautious. 


\section{Disinfection and isolation measures for biopsy operation}

\section{Environment and material}

Confirmed or suspected patients must be placed in isolation wards for preoperative preparation. Also, the operations should be performed in a separate operating room with negative pressure, or with an independent purification airconditioning unit.

As bronchoscopes enter the passage of the human body and contact with the luminal mucosa, they need to reach a high level of disinfection with regular biological monitoring. Moreover, every time after operation, the operator should press the suction button to suck out the mucus and blood remained in the biopsy channel with clean water, and then send bronchoscopies to the cleaning room for decontamination and disinfection.

\section{Operators and patients}

The first principle to avoid cross-infection is to minimize the number of people involved in the operation. Meanwhile doctors and nurses involved need to implement protective measures of biosafety level 3 with disposable surgical caps, medical protective masks, medical protective face masks (or full facepiece respirators or positive pressure headgear), protective clothing, disposable latex gloves, and disposable shoe covers. During the operation, patients should also wear masks to reduce or prevent the splashing of secretions.

\section{Postoperative management}

The obtained specimens need to be placed in a special closed container with special symbols. The inspectors should wear double-layer gloves during inspection. The operating room should be thoroughly disinfected after the operation, and can be reused after passing the sampling test of the infection management department.

\section{Differential diagnosis with complications after biopsy operation}

The primary complications that arise with TBB or CTguided percutaneous thoracic biopsy are pneumothorax and hemorrhage, whose symptoms include chest pain, hemoptysis, and shortness of breath after the procedure (59). The chest radiography is currently recommended for patients who become symptomatic after the procedure of biopsy operation.

The diagnosis of pneumothorax can be easily set up and distinguished from COVID-19 by chest X-ray. The $\mathrm{X}$-ray images of pneumothorax always show the pleural line, which is the outer margin of the visceral pleura (and lung) and is separated from the pleura (and chest wall) by a lucent gas space devoid of pulmonary vessels (60). CT is the first choice in detecting the presence of a suspected pulmonary hemorrhage, by resenting a focal patchy alveolar infiltration, which is worth identifying with COVID-19 (61). However, hemoptysis together with normal body temperature and WBC count can help distinguish pulmonary hemorrhage.

\section{Correlation between COVID-19 and lung cancers}

A latest study revealed the correlation between COVID-19 and cancers (62). Among the included patients of COVID-19, about $1 \%$ patients had a history of cancer, which is higher than the incidence of cancer in China, at $0.29 \%$. Furthermore, lung cancer is the most frequent of all cancers in COVID-19 patients. Importantly, cancer patients are faced with a higher risk of developing a severe case of COVID-19 than non-cancer patients, verified with a logistic regression and a Cox regression model.

Therefore, the research team proposed three major strategies for cancer patients in the epidemic of COVID-19, which we should apply in clinical work and inform cancer patients. First, when oncologists perform the chemotherapy and surgery, more care should be taken in the disease endemic area. Second, patients with a history of cancer should strengthen personal protection. Third, we should strengthen monitoring and give priority to the patients who infected with SARS-CoV-2 with a history of cancer, especially the elderly and those with complications (62).

\section{Summary}

As a thoracic oncologist, there are varies of new challenges, faced with the epidemic of COVID-19. At outpatient, we need to distinguish COVID-19 from other lung diseases based on symptoms and signs, such as GGN of lung cancer, PTLC, PLC, interstitial pneumonia, PAP and other common infectious pneumonia. In the routine of oncology, the differential diagnosis of complications and adverse events from COVID-19 is also significant, including radiation pneumonitis, CIP, neutropenic fever, and lung toxicity of targeted therapy, cancer-associate bacterial 
pneumonia, and fever in cancer patients.

TBB and CT-guided percutaneous thoracic biopsy are the most important diagnostic operations in thoracic oncology. In the epidemic of COVID-19, to protect the safety of patients and medical staff, we need implement following measures: (I) screen suspected and confirmed patients before the operation; (II) implement disinfection and isolation measures during the operation; (III) differentiate common complications such as pneumothorax and hemorrhage from COVID-19 after the operation.

It is worth mentioning that, patients with cancers especially lung cancers are more susceptible to COVID-19 and are easier to occur sever cases once infection. To this end, we need to pay more attention to the protection of cancer patients.

\section{Acknowledgments}

Funding: This study was supported in part by a grant from National Natural Science Foundation of China (81802255), Shanghai Pujiang Program (17PJD036) and a grant from Shanghai Municipal Commission of Health and Family Planning Program (20174Y0131), National key research \& development project (2016YFC0902300), Major disease clinical skills enhancement program of three year action plan for promoting clinical skills and clinical innovation in municipal hospitals, Shanghai Shen Kang Hospital Development Center Clinical Research Plan of SHDC (16CR1001A), "Dream Tutor" Outstanding Young Talents Program (fkyq1901), key disciplines of Shanghai Pulmonary Hospital (2017ZZ02012), grant of Shanghai Science and Technology Commission (16JC1405900).

\section{Footnote}

Conflicts of Interest: All authors have completed the ICMJE uniform disclosure form (available at http://dx.doi. org/10.21037/tlcr.2020.02.10). YH serves as the unpaid editorial board member of Translational Lung Cancer Research from Jan 2020 to Dec 2021. The other authors have no conflicts of interest to declare.

Ethical Statement: The authors are accountable for all aspects of the work in ensuring that questions related to the accuracy or integrity of any part of the work are appropriately investigated and resolved.

Open Access Statement: This is an Open Access article distributed in accordance with the Creative Commons Attribution-NonCommercial-NoDerivs 4.0 International License (CC BY-NC-ND 4.0), which permits the noncommercial replication and distribution of the article with the strict proviso that no changes or edits are made and the original work is properly cited (including links to both the formal publication through the relevant DOI and the license). See: https://creativecommons.org/licenses/by-nc-nd/4.0/.

\section{References}

1. Habibzadeh P, Stoneman EK. The Novel Coronavirus: A Bird's Eye View. Int J Occup Environ Med 2020;11:65-71.

2. Yin Y, Wunderink RG. MERS, SARS and other coronaviruses as causes of pneumonia. Respirology 2018;23:130-7.

3. Chen N, Zhou M, Dong X, et al. Epidemiological and clinical characteristics of 99 cases of 2019 novel coronavirus pneumonia in Wuhan, China: a descriptive study. Lancet 2020;395:507-13.

4. Akinosoglou KS, Karkoulias K, Marangos M. Infectious complications in patients with lung cancer. Eur Rev Med Pharmacol Sci 2013;17:8-18.

5. Gardner JG, Bhamidipati DR, Rueda AM, et al. White Blood Cell Counts, Alcoholism, and Cirrhosis in Pneumococcal Pneumonia. Open Forum Infect Dis 2017;4:ofx034.

6. Galván JM, Rajas O, Aspa J. Review of Non-Bacterial Infections in Respiratory Medicine: Viral Pneumonia. Arch Bronconeumol 2015;51:590-7.

7. Lee KY, Youn YS, Lee JW, et al. Mycoplasma pneumoniae pneumonia, bacterial pneumonia and viral pneumonia. J Pediatr (Rio J) 2010;86:448-50.

8. Daxboeck F, Krause R, Wenisch C. Laboratory diagnosis of Mycoplasma pneumoniae infection. Clin Microbiol Infect 2003;9:263-73.

9. Ruuskanen O, Lahti E, Jennings LC, et al. Viral pneumonia. Lancet 2011;377:1264-75.

10. Hansell DM, Bankier AA, MacMahon H, et al. Fleischner Society: glossary of terms for thoracic imaging. Radiology 2008;246:697-722.

11. Migliore M, Fornito M, Palazzolo M, et al. Ground glass opacities management in the lung cancer screening era. Ann Transl Med 2018;6:90.

12. Chung $M$, Bernheim A, Mei X, et al. CT Imaging Features of 2019 Novel Coronavirus (2019-nCoV). Radiology 2020. doi: 10.1148/radiol.2020200236.

13. Song F, Shi N, Shan F, et al. Emerging Coronavirus 
2019-nCoV Pneumonia. Radiology 2020. doi: 10.1148/ radiol.2020200274.

14. Morikawa S, Okamura T, Yamaguchi T, et al. Clinical features of primary lung cancer presenting as pulmonary consolidation mimicking pneumonia. Fujita Medical Journal 2016;2:17-21.

15. Truong MT, Ko JP, Rossi SE, et al. Update in the evaluation of the solitary pulmonary nodule. Radiographics 2014;34:1658-79.

16. Martínez-Jiménez S, Rosado-de-Christenson ML, Walker $\mathrm{CM}$, et al. Imaging features of thoracic metastases from gynecologic neoplasms. Radiographics 2014;34:1742-54.

17. Ikezoe J, Godwin JD, Hunt KJ, et al. Pulmonary lymphangitic carcinomatosis: chronicity of radiographic findings in long-term survivors. AJR Am J Roentgenol 1995; 165:49-52.

18. Johkoh T, Ikezoe J, Tomiyama N, et al. CT findings in lymphangitic carcinomatosis of the lung: correlation with histologic findings and pulmonary function tests. AJR Am J Roentgenol 1992;158:1217-22.

19. Munk PL, Müller N, Miller RR, et al. Pulmonary lymphangitic carcinomatosis: CT and pathologic findings. Radiology 1988;166:705-9.

20. Fang Y, Zhang H, Xu Y, et al. CT Manifestations of Two Cases of 2019 Novel Coronavirus (2019-nCoV) Pneumonia. Radiology 2020;295:208-9.

21. De Wever W, Meersschaert J, Coolen J, et al. The crazypaving pattern: a radiological-pathological correlation. Insights Imaging 2011;2:117-32.

22. Bouros D, Nicholson AC, Polychronopoulos V, et al. Acute interstitial pneumonia. Eur Respir J 2000;15:412-8.

23. Mo Q, Wang B, Dong N, et al. The Clinical Clues of Pulmonary Alveolar Proteinosis: A Report of 11 Cases and Literature Review. Can Respir J 2016;2016:4021928.

24. Benveniste MF, Gomez D, Carter BW, et al. Recognizing Radiation Therapy-related Complications in the Chest. Radiographics 2019;39:344-66.

25. Bledsoe TJ, Nath SK, Decker RH. Radiation Pneumonitis. Clin Chest Med 2017;38:201-8.

26. Liu Y, Wang H, Deng J, et al. Toxicity of tumor immune checkpoint inhibitors-more attention should be paid. Transl Lung Cancer Res 2019;8:1125-33.

27. Chuzi S, Tavora F, Cruz M, et al. Clinical features, diagnostic challenges, and management strategies in checkpoint inhibitor-related pneumonitis. Cancer Manag Res 2017;9:207-13.

28. Shohdy KS, Abdel-Rahman O. Neurological complications of anti-PD-1 antibodies: shall we be more concerned?
Transl Cancer Res 2018;7:S436-8.

29. Higenbottam T, Kuwano K, Nemery B, et al. Understanding the mechanisms of drug-associated interstitial lung disease. Br J Cancer 2004;91 Suppl 2:S31-7.

30. Matsumoto Y, Kawaguchi T, Yamamoto N, et al. Interstitial Lung Disease Induced by Osimertinib for Epidermal Growth Factor Receptor (EGFR) T790Mpositive Non-small Cell Lung Cancer. Intern Med 2017;56:2325-8.

31. Klastersky JA. Adverse events of targeted therapies. Curr Opin Oncol 2014;26:395-402.

32. Rolston KV. Postobstructive Pneumonia in Cancer Patients. Clin Infect Dis 2016;63:707-8.

33. Kalkat MS, Bonser RS. Obstructive pneumonia: an indication for surgery in mega aorta syndrome. Ann Thorac Surg 2003;75:1313-5.

34. Waheed Z, Irfan M, Fatimi S, et al. Bronchial carcinoid presenting as multiple lung abscesses. J Coll Physicians Surg Pak 2013;23:229-30.

35. Kohno S, Koga H, Oka M, et al. The pattern of respiratory infection in patients with lung cancer. Tohoku J Exp Med 1994;173:405-11.

36. Valvani A, Martin A, Devarajan A, et al. Postobstructive pneumonia in lung cancer. Ann Transl Med 2019;7:357-.

37. Boiselle PM, Ernst A. Recent Advances in Central Airway Imaging. Chest 2002;121:1651-60.

38. Finkelstein SE, Schrump DS, Nguyen DM, et al. Comparative evaluation of super high-resolution CT scan and virtual bronchoscopy for the detection of tracheobronchial malignancies. Chest 2003;124:1834-40.

39. Jain P, Sandur S, Meli Y, et al. Role of flexible bronchoscopy in immunocompromised patients with lung infiltrates. Chest 2004;125:712-22.

40. Safdar A, Armstrong D. Infectious morbidity in critically ill patients with cancer. Crit Care Clin 2001;17:531-70.

41. Joos L, Tamm M. Breakdown of pulmonary host defense in the immunocompromised host: cancer chemotherapy. Proc Am Thorac Soc 2005;2:445-8.

42. Lal A, Bhurgri Y, Rizvi N, et al. Factors influencing inhospital length of stay and mortality in cancer patients suffering from febrile neutropenia. Asian Pac J Cancer Prev 2008;9:303-8.

43. Ashour HM, el-Sharif A. Microbial spectrum and antibiotic susceptibility profile of gram-positive aerobic bacteria isolated from cancer patients. J Clin Oncol 2007;25:5763-9.

44. Charles PE, Ladoire S, Aho S, et al. Serum procalcitonin 
elevation in critically ill patients at the onset of bacteremia caused by either Gram negative or Gram positive bacteria. BMC Infect Dis 2008;8:38.

45. Crawford J, Dale DC, Lyman GH. Chemotherapyinduced neutropenia: risks, consequences, and new directions for its management. Cancer 2004;100:228-37.

46. Freifeld AG, Bow EJ, Sepkowitz KA, et al. Clinical practice guideline for the use of antimicrobial agents in neutropenic patients with cancer: 2010 update by the infectious diseases society of america. Clin Infect Dis 2011;52:e56-93.

47. White L, Ybarra M. Neutropenic Fever. Hematol Oncol Clin North Am 2017;31:981-93.

48. Mackowiak PA, LeMaistre CF. Drug fever: a critical appraisal of conventional concepts. An analysis of 51 episodes in two Dallas hospitals and 97 episodes reported in the English literature. Ann Intern Med 1987;106:728-33.

49. Kimura H, Matsui Y, Ishikawa A, et al. Randomized controlled phase III trial of adjuvant chemoimmunotherapy with activated killer $\mathrm{T}$ cells and dendritic cells in patients with resected primary lung cancer. Cancer Immunol Immunother 2015;64:51-9.

50. Ogawara D, Fukuda M, Ueno S, et al. Drug fever after cancer chemotherapy is most commonly observed on posttreatment days 3 and 4. Support Care Cancer 2016;24:615-9.

51. Chang JC. How to differentiate neoplastic fever from infectious fever in patients with cancer: usefulness of the naproxen test. Heart Lung 1987;16:122-7.

52. Blay JY, Negrier S, Combaret V, et al. Serum level of interleukin 6 as a prognosis factor in metastatic renal cell carcinoma. Cancer Res 1992;52:3317-22.

53. Theologides A. Generalized perturbations in host physiology caused by localized tumors. The anorexia-

Cite this article as: Guo H, Chen X, Su C, Liu Y, Wang H, Sun C, Chen P, Jiang M, Xu Y, Wu S, Jia K, Zhao S, Li W, Chen B, Wang L, Yu J, Xiong A, Gao G, Wu F, Li J, Ye L, Bo B, Chen S, Ren S, He Y, Zhou C. Challenges and countermeasures of thoracic oncology in the epidemic of COVID-19. Transl Lung Cancer Res 2020;9(2):337-347. doi: 10.21037/tlcr.2020.02.10 cachexia syndrome: a new hypothesis. Ann N Y Acad Sci 1974;230:14-22.

54. Zell JA, Chang JC. Neoplastic fever: a neglected paraneoplastic syndrome. Support Care Cancer 2005;13:870-7.

55. Remiszewski P, Slodkowska J, Wiatr E, et al. Fatal infection in patients treated for small cell lung cancer in the Institute of Tuberculosis and Chest Diseases in the years 1980-1994. Lung Cancer 2001;31:101-10.

56. Shahid M, Malik A, Bhargava R. Bronchogenic carcinoma and secondary aspergillosis--common yet unexplored: evaluation of the role of bronchoalveolar lavage-polymerase chain reaction and some nonvalidated serologic methods to establish early diagnosis. Cancer 2008;113:547-58.

57. Akinosoglou KS, Karkoulias K, Marangos M. Infectious complications in patients with lung cancer. Eur Rev Med Pharmacol Sci 2013;17:8-18.

58. Wilkin A, Feinberg J. Pneumocystis carinii pneumonia: a clinical review. Am Fam Physician 1999;60:1699-708, 1713-4.

59. Shofer S, Wahidi MM. Transbronchial Lung Biopsy. In: Ernst A, Herth FJF, editors. Introduction to Bronchoscopy. 2 ed. Cambridge: Cambridge University Press; 2017:118-28.

60. O'Connor AR, Morgan WE. Radiological review of pneumothorax. BMJ 2005;330:1493-7.

61. Marasco WJ, Fishman EK, Kuhlman JE, et al. Acute pulmonary hemorrhage. CT evaluation. Clin Imaging 1993;17:77-80.

62. Liang W, Guan W, Chen R, et al. Cancer patients in SARS-CoV-2 infection: a nationwide analysis in China. Lancet Oncol 2020. DOI: 10.1016/S14702045(20)30096-6. 